PROCEEDINGS OF THE

AMERICAN MATHEMATICAL SOCIETY

Volume 124, Number 6, June 1996

\title{
A COUNTEREXAMPLE TO THE DIFFERENTIABILITY OF THE BERGMAN KERNEL FUNCTION
}

\author{
SO-CHIN CHEN
}

(Communicated by Eric Bedford)

\begin{abstract}
In this paper we prove the following main result. Let $D$ be a smoothly bounded pseudoconvex domain in $\mathbf{C}^{n}$ with $n \geq 2$. Suppose that there exists a complex variety sitting in the boundary $b D$; then we have

$$
K_{D}(z, w) \notin C^{\infty}(\bar{D} \times \bar{D}-\Delta(b D)) .
$$

In particular, the Bergman kernel function associated with the DiederichFornaess worm domain is not smooth up to the boundary in joint variables off the diagonal of the boundary.
\end{abstract}

\section{INTRODUCTION}

In several complex variables it has been shown that the Bergman kernel function is closely related to the boundary regularity of holomorphic mappings between two domains. Therefore, it is always a fundamental question to investigate the boundary regularity of the Bergman kernel function associated with a smoothly bounded pseudoconvex domain.

Historically, it was first proved by Kerzman [10], based on Kohn's work, that the Bergman kernel function associated to a smoothly bounded strictly pseudoconvex domain $D$ can be extended smoothly to $\bar{D} \times \bar{D}-\Delta(b D)$, where $\Delta(b D)=\{(z, z) \mid z \in$ $b D\}$. Later it was generalized independently by Bell [2] and Boas [3] to the following.

Theorem 1.1. Let $D$ be a smoothly bounded pseudoconvex domain in $\mathbf{C}^{n}$. If $\Gamma_{1}$ and $\Gamma_{2}$ are disjoint open subsets of $b D$ consisting of points of finite type in the sense of D'Angelo [8], then the Bergman kernel function associated to D extends smoothly to $\Gamma_{1} \times \Gamma_{2}$.

Theorem 1.2. Let $D$ be a smoothly bounded pseudoconvex domain in $\mathbf{C}^{n}$. Suppose that condition $R$ holds on $D$. Let $\Gamma_{1}$ and $\Gamma_{2}$ be two disjoint open subsets of $b D$ such that $\Gamma_{1}$ consists of points of finite type. Then the Bergman kernel function associated to $D$ extends smoothly to $\Gamma_{1} \times \Gamma_{2}$.

Here condition $R$ means that the Bergman projection $P$, the orthogonal projection from $L^{2}(D)$ onto the closed subspace $H^{2}(D)$ of square-integrable holomorphic functions, maps $C^{\infty}(\bar{D})$ continuously into itself.

Based on the above theorems it is natural to conjecture that a similar extension phenomenon might probably hold on any smoothly bounded pseudoconvex domain in $\mathbf{C}^{n}$. However, in this article we are going to prove the following main result.

Received by the editors December 1, 1994.

1991 Mathematics Subject Classification. Primary 32H10. 
Main Theorem. Let $D$ be a smoothly bounded pseudoconvex domain in $\mathbf{C}^{n}$ with $n \geq 2$. Suppose that there exists a complex variety sitting in the boundary bD; then we have

$$
K_{D}(z, w) \notin C^{\infty}(\bar{D} \times \bar{D}-\Delta(b D)),
$$

where $\Delta(b D)=\{(z, z) \mid z \in b D\}$.

\section{Two EXAMPLES}

In this section we will present two examples. First we fix a smooth real-valued function $\lambda: \mathbf{R} \rightarrow \mathbf{R}$ as in [9] with the following properties:

(i) $\lambda(x)=0$ if $x \leq 0$.

(ii) $\lambda(x)>0$ if $x>1$.

(iii) $\lambda^{\prime \prime}(x) \geq 100 \lambda^{\prime}(x)$ for all $x$.

(iv) $\lambda^{\prime \prime}(x)>0$ if $x>0$.

(v) $\lambda^{\prime}(x)>100$ if $\lambda(x)>\frac{1}{2}$.

Example A. For any $r>1$, define the domain $\Omega_{r}$ by

$$
\Omega_{r}=\left\{(z, w) \in \mathbf{C}^{2} \mid \rho_{r}(z, w)<0\right\},
$$

where $\rho_{r}(z, w)=|z|^{2}-1+\lambda\left(|w|^{2}-r^{2}\right)$.

Then we have

Lemma 2.1. The domain $\Omega_{r}$ defined as above is a smoothly bounded pseudoconvex domain in $\mathbf{C}^{2}$ satisfying that

(a) $\Omega_{r}$ is convex.

(b) Condition $R$ holds on $\Omega_{r}$.

(c) $\Omega_{r}$ is strictly pseudoconvex everywhere except on the set

$$
M_{r}=\left\{(z, w) \in \mathbf{C}^{2}|| z \mid=1 \text { and } 0 \leq|w| \leq r\right\}
$$

Condition $R$ on $\Omega_{r}$ follows from either (a) or the fact that $\Omega_{r}$ is Reinhardt. For instance, see Chen [6], [7], Boas and Straube [4] or Straube [12]. Obviously, the boundary contains a family of complex discs parametrized by the unit circle. Hence by our main theorem we have

$$
K_{\Omega_{r}}(z, w) \notin C^{\infty}\left(\bar{\Omega}_{r} \times \bar{\Omega}_{r}-\Delta\left(b \Omega_{r}\right)\right) .
$$

But we would like to provide an elementary proof of the main theorem for $\Omega_{r}$ here which gives a more geometric insight into the problem.

Let $\Delta_{r}=\left\{w \in \mathbf{C}|| w \mid<\sqrt{r^{2}+1}\right\}$, and denote by $\Delta$ the unit disc in the complex plane. Put $D_{r}=\Delta \times \Delta_{r}$. $D_{r}$ is a pseudoconvex domain in $\mathbf{C}^{2}$, and obviously we have $\Omega_{r} \subseteq D_{r}$. It is also clear that $\Lambda=\left\{z^{m} w^{n} \mid m, n \in \mathbf{N} \cup\{0\}\right\}$ forms a complete orthogonal basis for both $H^{2}\left(\Omega_{r}\right)$ and $H^{2}\left(D_{r}\right)$. So we have

$$
c_{m n}^{r}=\int_{\Omega_{r}}\left|z^{m} w^{n}\right|^{2} d \sigma \leq \int_{D_{r}}\left|z^{m} w^{n}\right|^{2} d \sigma=d_{m n}^{r} \text { for all } m, n .
$$

Both equalities are definitions. Now consider any point sequence $\left\{z_{j}\right\}_{j=1}^{\infty}$ in $\Delta$ such that $\left\{z_{j}\right\}$ approaches a boundary point, say $z_{0}$. Pick any two positive numbers $p$ and $q$ such that $0<p<q<r$. So now we have two point sequences $\left\{\left(z_{j}, p\right)\right\}$ and $\left\{\left(z_{j}, q\right)\right\}$ in both $\Omega_{r}$ and $D_{r}$ which approach the boundary points $\left(z_{0}, p\right)$ and $\left(z_{0}, q\right)$ respectively. Clearly we have $\left(z_{0}, p\right) \neq\left(z_{0}, q\right)$. Next, by using the explicit formula 
for the Bergman kernel function $K_{\Delta}(z, w)$ on the unit disc in the complex plane, we obtain

$$
\begin{aligned}
K_{\Omega_{r}}\left(\left(z_{j}, p\right),\left(z_{j}, q\right)\right) & =\sum_{m=0}^{\infty} \sum_{n=0}^{\infty} \frac{1}{c_{m n}^{r}}\left|z_{j}\right|^{2 m} \cdot(p q)^{n} \\
& \geq \sum_{m=0}^{\infty} \sum_{n=0}^{\infty} \frac{1}{d_{m n}^{r}}\left|z_{j}\right|^{2 m} \cdot(p q)^{n} \\
& =K_{\Delta}\left(z_{j}, z_{j}\right) K_{\Delta_{r}}(p, q) \\
& =\frac{1}{\pi} \cdot \frac{1}{\left(1-\left|z_{j}\right|^{2}\right)^{2}} K_{\Delta_{r}}(p, q) .
\end{aligned}
$$

It follows that

$$
\lim _{j \rightarrow \infty} K_{\Omega_{r}}\left(\left(z_{j}, p\right),\left(z_{j}, q\right)\right)=\infty
$$

Hence the Bergman kernel function $K_{\Omega_{r}}(z, w)$ is not bounded near $z=\left(z_{0}, p\right)$ and $w=\left(z_{0}, q\right)$. In particular, it shows that $K_{\Omega_{r}}(z, w) \notin C^{\infty}\left(\bar{\Omega}_{r} \times \bar{\Omega}_{r}-\Delta\left(b \Omega_{r}\right)\right)$. This completes the proof of the main theorem on $\Omega_{r}$.

Example B (Diederich-Fornaess worm domain). Now define the domain $\Omega_{r}$, for any $r>1$, by

$$
\Omega_{r}=\left\{(z, w) \in \mathbf{C}^{2} \mid \rho_{r}(z, w)<0\right\}
$$

where $\rho_{r}(z, w)=\left|z+\exp \left(i \ln |w|^{2}\right)\right|^{2}-1+\lambda\left(\frac{1}{|w|^{2}}-1\right)+\lambda\left(|w|^{2}-r^{2}\right)$. Then we obtain the famous Diederich-Fornaess worm domain [9]. These domains $\Omega_{r}$ s are smoothly bounded pseudoconvex in $\mathbf{C}^{2}$ and are strictly pseudoconvex everywhere except on the annulus

$$
A_{r}=\left\{(z, w) \in \mathbf{C}^{2} \mid z=0 \text { and } 1 \leq|w| \leq r\right\} .
$$

Therefore, again by our main theorem we see that

$$
K_{\Omega_{r}}(z, w) \notin C^{\infty}\left(\bar{\Omega}_{r} \times \bar{\Omega}_{r}-\Delta\left(b \Omega_{r}\right)\right) .
$$

We should point out that it has been shown in Barrett [1] that the Bergman projection on these domains does not preserve the Sobolev $k$-space $W^{k}\left(\Omega_{r}\right)$ if $k$ is large enough. However, it is still not known whether the condition $R$ holds on $\Omega_{r}$ or not.

\section{Proof of the MAIN TheOREM}

Let $D$ be a smoothly bounded pseudoconvex domain in $\mathbf{C}^{n}$ with $n \geq 2$, and let $V$ be a complex variety sitting in the boundary $b D$. Let $p \in V$ be a regular point of $V$. Denote by $\vec{n}$ the unit outward normal at $p$. Since the boundary of $D$ is smooth, there exists small $\delta, \varepsilon_{0}>0$ such that $w-\varepsilon \vec{n} \in D$ for all $w \in b D \cap B(p ; \delta)$ and all $0<\varepsilon<\varepsilon_{0}$. Now let us consider a small complex disc $\bar{\Delta} \subseteq b D \cap B(p ; \delta) \cap V$ centered at $p$. More precisely, $\Delta$ is the holomorphic embedding of the unit disc in the complex plane in the boundary, and the origin is mapped to $p$.

Suppose now that $K_{D}(z, w) \in C^{\infty}(\bar{D} \times \bar{D}-\Delta(b D))$. Then we have

$$
\sup _{w \in b \Delta}\left|K_{D}(p, w)\right| \leq M<+\infty,
$$

for some $M>0$. On the other hand, it was proved in P. Pflug [11] that

$$
\lim _{\varepsilon \rightarrow 0} K_{D}(p-\varepsilon \vec{n}, p-\varepsilon \vec{n})=+\infty .
$$


Then by the maximum modulus principle we get

$$
\sup _{w \in b \Delta_{\varepsilon}}\left|K_{D}(p-\varepsilon \vec{n}, w)\right| \geq K_{D}(p-\varepsilon \vec{n}, p-\varepsilon \vec{n}),
$$

where $\Delta_{\varepsilon}=\Delta-\varepsilon \vec{n} \subseteq D$. It follows that

$$
\sup _{w \in b \Delta}\left|K_{D}(p, w)\right|=\lim _{\varepsilon \rightarrow 0} \sup _{w \in b \Delta_{\varepsilon}}\left|K_{D}(p-\varepsilon \vec{n}, w)\right|=+\infty .
$$

This gives the desired contradiction, and the proof of the main theorem is now completed.

\section{Concluding REMARK}

In view of the main theorem proved above, it is natural to ask: How does the Bergman kernel function $K_{D}(z, w)$ behave in joint variables near the boundary, if the domain $D$ satsfies property $(P)$ introduced in Catlin [5]? So far we have no clue to answer this question.

\section{REFERENCES}

1. D. Barrett, Behavior of the Bergman projection on the Diederich-Fornaess worm, Acta Math., 168, (1992), 1-10. MR 93c:32033

2. S. Bell, Differentiability of the Bergman kernel and pseudo-local estimates, Math. Z., 192, (1986), 467-472. MR 87i:32034

3. H. P. Boas, Extension of Kerzman's theorem on differentiability of the Bergman kernel function, Indiana Univ. Math. J., 36, (1987), 495-499. MR 88j:32028

4. H. P. Boas and E. J. Straube, Sobolev estimates for the $\bar{\partial}-$ Neumann operator on domains in $\mathbf{C}^{n}$ admitting a defining function that is plurisubharmonic on the boundary, Math. Z., 206, (1991), 81-88. MR 92b:32027

5. D. Catlin, Global regularity for the $\bar{\partial}-$ Neumann problem, Proc. Sympos. Pure Math., vol. 41, Amer. Math. Soc., Providence, RI, 1984. MR 85j:32033

6. S. C. Chen, Global regularity of the $\bar{\partial}$-Neumann problem on circular domains, Math. Ann., 285, (1989), 1-12. MR 90i:32028

7. Global regularity of the $\bar{\partial}-$ Neumann problem in dimension two, Proc. Sympos. Pure Math., vol. 52, Part III, Amer. Math. Soc., Providence, RI, 1991, 55-61. MR 92h:32027

8. J. D'Angelo, Real hypersurfaces, order of contact, and applications, Ann. of Math., 115, (1982), 615-637. MR 84a:32027

9. K. Diederich and J. E. Fornaess, Pseudoconvex domains: an example with nontrivial Nebenhülle, Math. Ann., 225, (1977), 275-292. MR 55:3320

10. N. Kerzman, The Bergman kernel function. Differentiability at the boundary, Math. Ann., 195, (1972), 149-158. MR 45:3762

11. P. Pflug, Quadratintegrable holomorphe Funktionen und die Serre-Vermutung, Math. Ann., 216, (1975), 285-288. MR 52:3599

12. E. J. Straube, Exact regularity of Bergman, Szegö and Sobolev space projections in non pseudoconvex domains, Math. Z., 192, (1986), 117-128. MR 87k:32045

Institute of Applied Mathematics, National Tsing Hua University, Hsinchu 30043, Taiwan, Republic of China

E-mail address: scchen@am.nthu.edu.tw 